\title{
Evaluación y mejora en la información de los pacientes que se realizan una endoscopia
}

\author{
R. BAÑOS MADRID, T. RAMÓN ESPARZA', A. SERRANO JIMÉNEZ, \\ M. ALAJARÍN CERVERA, F. ALBERCA DE LAS PARRAS, J. MOLINA MARTÍNEZ \\ Unidad de Endoscopia Digestiva. Hospital Universitario Virgen de la Arrixaca. ${ }^{~}$ Técnico \\ Evaluación de Calidad. Murcia
}

EVALUATION AND IMPROVEMENT IN THE INFORMATION OF THE PATIENTS WHO CARRY OUT A DIGESTIVE ENDOSCOPY

\section{RESUMEN}

Introducción: La información es una parte importante del proceso asistencial, aunque en ocasiones puede ser inadecuada o deficiente.

Objetivo: Detectamos una oportunidad de mejora en la inadecuada información de los pacientes que se realizan una endoscopia. El objetivo es evaluar y mejorar la calidad de la información.

Método: Realizamos una primera evaluación con 100 pacientes procedentes de consultas, detectando desconocimiento de la técnica que se les va a realizar. Se aplican acciones de mejora, durante 4 meses, para realizar una segunda evaluación, con el mismo número de pacientes y evaluar mejoría respecto a la situación de partida.

Resultados: Se ha detectado un déficit de información en el momento de indicar la endoscopia (poca información aportada por el médico prescriptor y en un número importante de casos no se aportaba consentimiento informado) y tras realizar la prueba, no aportando recomendaciones. Priorizamos nuestra actuación a este nivel con medidas de mejora y después de una nueva evaluación se determina una mejora significativa en estas situaciones.

Conclusiones: Los estudios de nivel de calidad nos permiten detectar situaciones deficitarias y mejorar situaciones en los servicios de salud.

PALABRAS CLAVE: Evaluación. Calidad asistencial. Información. Endoscopia.

\begin{abstract}
Introduction: The information is an important part of the attendance process though in occasions it can be inadequate or deficient.

Aim: We detect an opportunity of improvement in the inadequate information of the patients who carry out an endoscopia. The aim is to evaluate and to improve the quality of the information.

Method: We realize the first evaluation with 100 patients proceeding from consultations, detecting ignorance of the technology. Actions of improvement are applied, for 4 months, to realize the second evaluation, with the same number of patients and to evaluate improvement with regard to the situation of item.

Results: A deficit of information has been detected in the moment to indicate the endoscopia (little information contributed by the doctor prescriptor and in an important number of cases informed assent was not contributed) and after realizing the test, not contributing recommendations. We prioritize our action to this level with measures of improvement and after a new evaluation, a significant improvement decides in these situations.

Conclusions: The studies of qualit level allow us to detect deficit situations and to improve situations in the services of health.
\end{abstract}

KEY WORDS: Evaluation. Attendance quality. Information. Endoscopia.

Baños Madrid R, Ramón Esparza T, Serrano Jiménez A, Alajarín Cervera M, Alberca de las Parras F, Molina Martínez J. Evaluación y mejora en la información de los pacientes que se realizan una endoscopia. An Med Interna (Madrid) 2005; 22: 413-418.

\section{INTRODUCCIÓN}

Actualmente la información es una parte importante de la práctica clínica, a todos nos parece lógico que el paciente tiene derecho a conocer que tipo de prueba diagnóstica y/o terapéutica va a realizarse, alternativas y complicaciones, y es a partir de esta información y en virtud del principio de autonomía, cuando el paciente podrá dar su consentimiento o no, para realizar una determinada actuación sanitaria. Afortuna- damente pasó a la historia, la actitud paternalista y la capacidad de decidir sin intervención del paciente, en la actualidad una determinada prueba diagnóstica o terapéutica debe ser consensuada entre médico y paciente, sobre la base de lo que es mejor para el paciente y esto solo se puede conseguir con una buena comunicación. Realizamos este estudio para valorar la información verbal y escrita que reciben los pacientes que se van a realizar una endoscopia digestiva de forma ambulatoria.

Trabajo aceptado: 25 de mayo de 2005 


\section{PACIENTES Y MÉTODO}

\section{DESCRIPCIÓN DE LA OPORTUNIDAD DE MEJORA}

Los pacientes que acuden a realizarse una endoscopia digestiva de forma ambulatoria tienen poca información sobre esta prueba, en ocasiones desconocen por que se la piden, alternativas a la prueba, complicaciones o incidencias que pueden pasar antes de la prueba -preparación para colonoscopia- durante su realización o posteriormente. Nuestro objetivo es evaluar la dimensión del problema en una primera evaluación, para posteriormente plantear una serie de medidas para mejorar la situación y en una segunda evaluación comparar los resultados con la primera y valorar si se ha mejorado. Es por tanto una autoevaluación que se realiza de forma prospectiva.

\section{DESCRIPCIÓN DE LOS COMPONENTES DEL ESTUDIO}

\section{Criterios a utilizar}

Criterio 1 (C1): El médico que solicita la prueba ha informado al paciente de las características de la misma.

Excepciones: No.

Aclaraciones: Tiene que informar como mínimo de porqué la pide, en que consiste, alternativas a la misma y complicaciones.

Criterio 2 (C2): Se proporciona información escrita antes de la endoscopia.

Excepciones: Si la citación es por teléfono.

Aclaraciones: Se tiene que informar del tipo de prueba, día, lugar de la cita, si se requiere preparación antes de la prueba.

Criterio 3 (C3): Se entrega documento de consentimiento informado cuando solicitan la endoscopia digestiva.

Excepciones: No.

Aclaraciones: Tiene que darse al paciente cuando se solicite la prueba en la consulta y el paciente debe entregarlo antes de realizarse la endoscopia.

Criterio 4 (C4): Existe consentimiento informado.

Excepciones: No.

Aclaraciones: Tiene que estar firmado por paciente y médico y quedar en la historia clínica.

Criterio 5 (C5): Se proporciona información tras la endoscopia por parte del médico que la hace.

Excepciones: Cuando se realiza con sedación, la información se dará al acompañante.

Aclaraciones: Se debe informar si se ha realizado terapeútica, complicaciones y recomendaciones tras la endoscopia.

Criterio 6 (C6): Se aportan documentos con recomendaciones tras la prueba.

Excepciones: Endoscopia diagnóstica.

Aclaraciones: Se deben entregar hojas con recomendaciones, en el caso de realizar endoscopias terapeúticas o sedación.

\section{Dimensiones a evaluar}

Se pretende evaluar la accesibilidad a la información verbal y escrita por parte del paciente antes y después de realizarse una endoscopia digestiva.

\section{Unidades de estudio}

Pacientes que acuden a realizarse una endoscopia digestiva, citados de forma ambulatoria, se aplica un cuestionario de recogida de datos (Tabla I). Se pretende evaluar la actividad del médico que solicita y del que realiza la prueba, teniendo en cuenta el momento cuando se solicita la prueba y cuando se realiza.

\section{TABLA I}

CUESTIONARIO DE RECOGIDA DE DATOS, UTILIZADO EN LA PRIMERA Y SEGUNDA EVALUACIÓN

El paciente ha recibido información del médico que pide la prueba (porque hay que hacerla, en que consiste o alternativas a la misma) sí

NO

Excepciones: No

El paciente tiene algún documento donde se especifique (prueba, día, lugar y tipo de preparación)

sí

NO

Excepciones: Que la citación se realice de forma telefónica

Se proporciona documento de consentimiento informado al solicitar la prueba en las consultas

sí NO

Excepciones: No

El documento de consentimiento informado está firmado por médico y paciente y está en la historia clínica

sí

$\mathrm{NO}$

Excepciones: No

Informa el médico que realiza la prueba de posibles complicaciones, recomendaciones, tras la prueba

sí NO

Excepciones: Si el paciente esté sedado, se dará esta información al acompañante

Se aporta algún documento tras la prueba con recomendaciones sí

NO

Excepciones: Cuando la endoscopia es diagnostica

\section{Identificación y muestreo de las unidades de estudio}

Decidimos evaluar a los pacientes citados para endoscopia digestiva desde el ambulatorio de especialidades de nuestro hospital, durante el mes de junio de 2003 (100 casos) -primera evaluación.

Como fuente de datos se utilizó al paciente y la historia clínica. El tamaño de la muestra fue igual al marco muestral, por lo que no fue necesario realizar ningún método de muestreo.

\section{Reevaluación}

Durante los meses de julio a octubre de 2003 se aplican las acciones de mejora (Tabla II), realizándose una segunda eva- 


\section{GUIAS DE LAAMSOCIACIÓN ESPAÑOLA DE CIRUJANOS
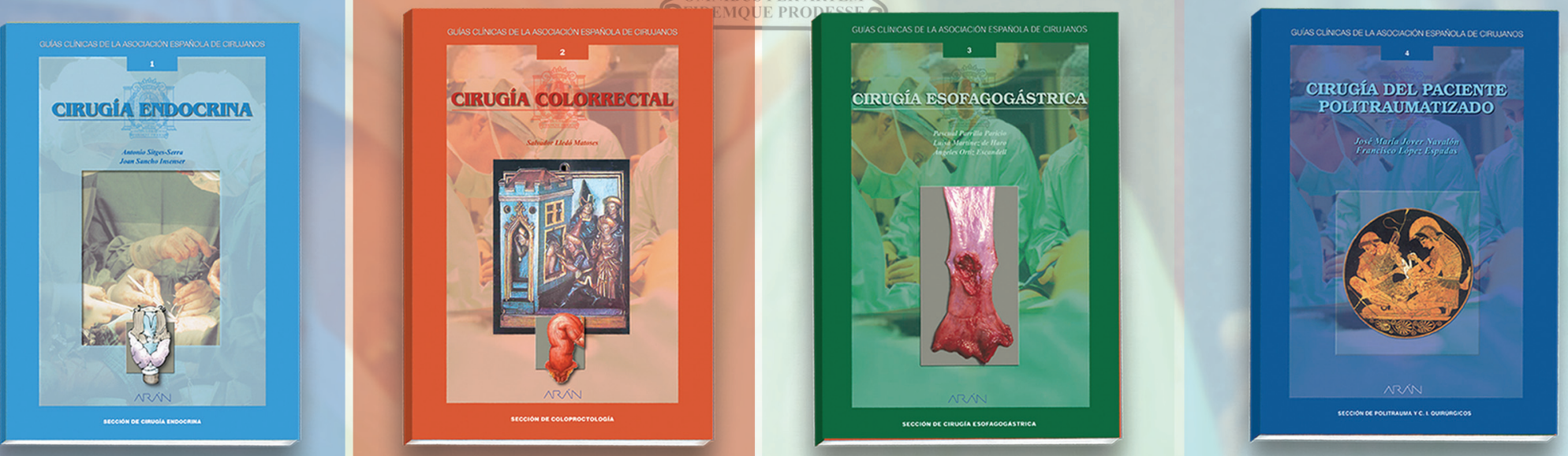
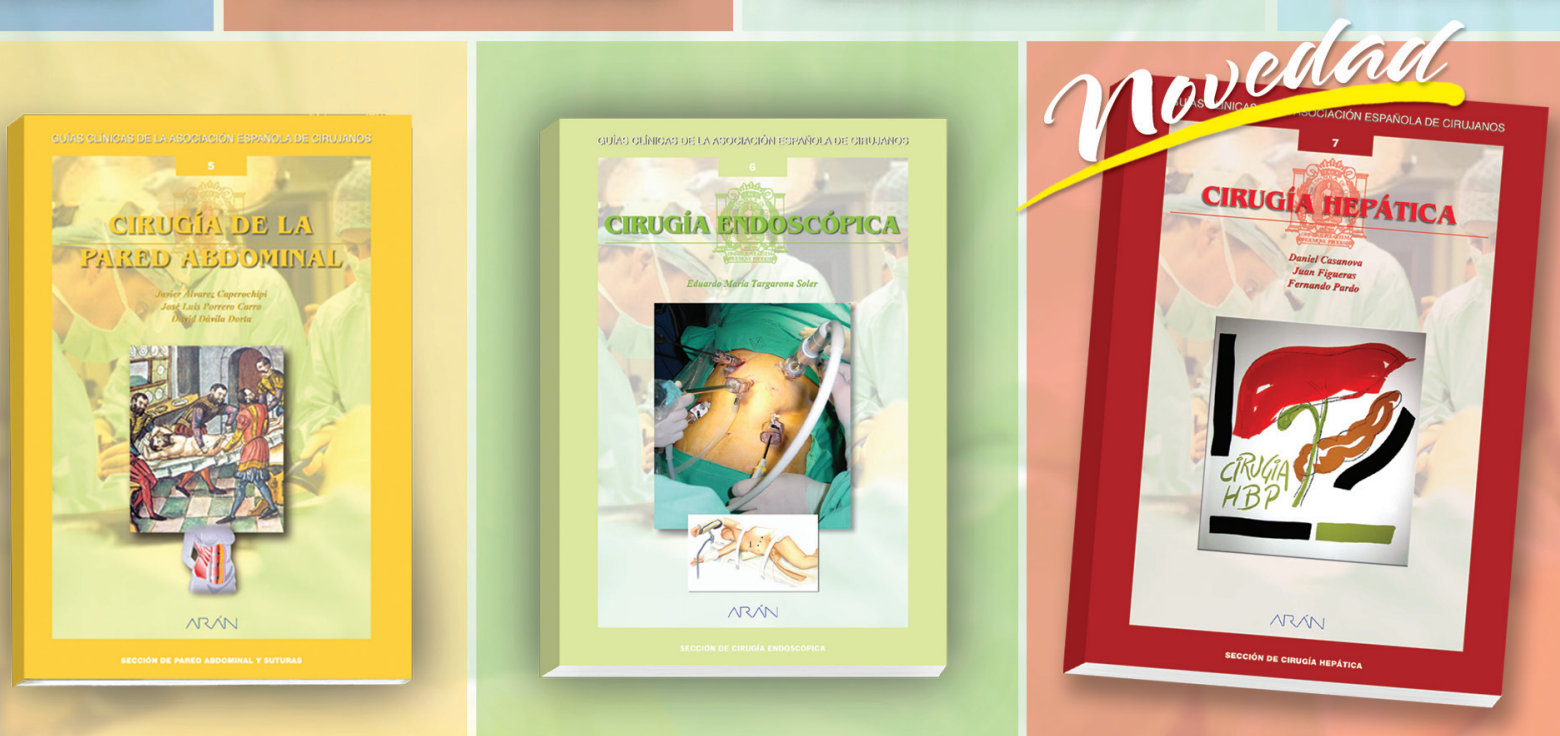

Deseo recibir los ejemplares marcados:

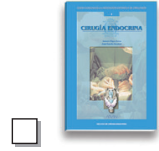

Cirugía

Endocrina

$18,03 €$ I.V.A. incluido

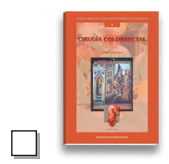

Cirugía

Colorrectal

$25 €$ I.V.A. incluido

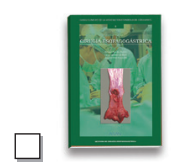

Cirugía

Esofagogástrica $28,13 €$ I.V.A. incluido

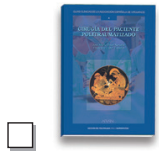

Cirugía del Paciente Politraumatizado $25 €$ I.V.A. incluido

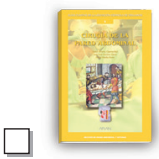

Cirugía de la Pared Abdominal $25 €$ I.V.A. incluido

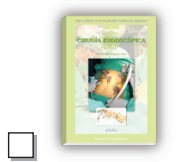

Cirugía Endoscópica $31,20 €$ I.V.A. incluido

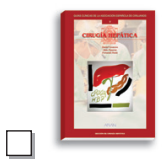

Cirugía Hepática $28,13 €$ I.V.A. incluido

Total $($.................. $)$

Apellidos y nombre

Dirección

Población Provincia

C. P.

Teléfono Fax e-mail

Especialidad Centro de trabajo

Forma de pago: Contra reembolso $\square$ Talón adjunto 

dos veces al dia. Grupos especiales de pacientes: No es necesario ajustar la dosis en pacientes ancianos o en aquéllos con insuficiencia renal. No se dispone de datos sobre el uso de Seretide en pacientes con insuficiencia hepática

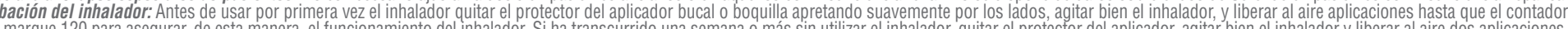
de dosis marque 120 para asegurar, de esta manera, el funcionamiento del inhalador. Si ha transcurrido una semana o más sin utilizar el inhalador, quitar el protector del aplicador, agitar bien el inhalador y liberar al aire dos aplicaciones.

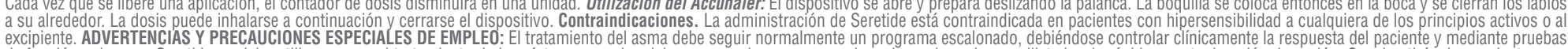
de función pulmonar. Seretide no debe utilizarse para el tratamiento de los síntomas agudos del asma para los que se requiere el uso de un broncodilatador de rápida y corta duración de acción. Se advertirá a los pacientes que dispongan en todo momento de su medicación para el alivio de sus síntomas en un ataque de asma agudo. Seretide no está dirigido al tratamiento inicial del asma hasta que se haya determinado la necesidad de corticosteroides y
una posología aproximada. La utilización creciente de broncodilatadores de corta duración de acción para aliviar los síntomas del asma indica un empeoramiento en el control del asma y los pacientes deberán ser examinados po

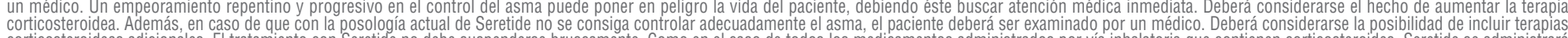
corticosteroideas adicionales. El tratamiento con Seretide no debe suspenderse bruscamente. Como en el caso de todos los medicamentos administrados por vía inhalatoria que contienen corticosteroides, Seretide se administrarará con precaución a pacientes con tuberculosis pulmonar. Seretide deberá administrarse con precaución a pacientes con problemas cardiovasculares graves, incluyendo anormalidades en el ritmo cardiaco, diabetes mellitus, hipopotasemia
no tratada o tirotoxicosis. El tratamiento sistémico con agonistas beta-2 puede producir una hipopotasemia potencialmente grave pero, los niveles plasmáticos de salmeterol son muy bajos tras la inhalación de dosis terapeuticas. Al no tratada o tirotoxicosis. El tratamiento sistemico con agonistas beta-2 puede producir una hipopotasemia potencialmente grave pero, los niveles plasmaticos de salmeterol son muy bajos tras la inhalacion de dosis terapeuticas. An de Seretide, examinarse al paciente e instituirse una terapia alternativa si fuera necesario. Debera tenerse cuidado cuando los pacientes pasen a recibir tratamiento con Seretide, particularmente si hubiera alguna razón para supone
que la función corticosuprarrenal está alterada a causa de un tratamiento previo con esteroides por vía sistémica. Pueden aparecer efectos sistémicos con cualquier corticosteroide administrado por vía inhalatatoria, especialmente a

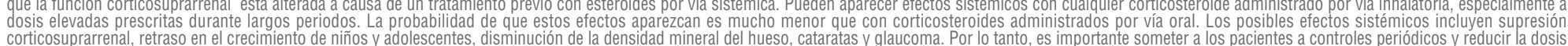
corticosuprarrenal, retraso en el crecimiento de niños y adolescentes, disminución de la densidad mineral del hueso, cataratas y glaucoma. Por lo tanto, es importante someter a los pacientes a controles periódicos y reducir a dosis
de corticosteroide inhalado a la dosis mínima con la que se mantenga un control eficaz del asma. Se recomienda controlar de forma regular la altura de los niños que reciben tratamiento prolongado con corticosteroides inhalados. El tratamiento prolongado con altas dosis de corticosteroides inhalados puede causar supresión corticosuprarrenal y crisis corticosuprarrenal aguda. Los niños y yadolescentes < 16 años que reciben dosis más altas que las permitidas de fluticasona (habitualmente $>1000 \mathrm{mcg} /$ día) puede estar, en particular, en situación de riesgo. Las situaciones que podrían potencialmente desencadenar una crisis corticosuprarrenal aguda, incluyen trauma, cirugía, infección o
cualquier reducción rápida de la dosis. Los síntomas que aparecen son habitualmente vagos y pueden incluir anorexia, dolor abdominal, pérdida de peso, cansancio cefalea náuseas vómitos, hipotensión, disminución del nivel de cualquier reducción rápida de la dosis. Los sintomas que aparecen son habitualmente vagos y pueden incluir anorexia, dolor abdominal, perdida de peso, cansancio, cefalea, náuseas, vómitos, hipotensión, disminucion del nivel de
consciencia, hipoglucemia y convulsiones. Se debe considerar la administración adicional de corticosteroides por vía sistémica durante periodos de estrés o cirugía electiva. Como la absorción sistémica tiene lugar en gran manera a través de los pulmones, el uso de un espaciador con el inhalador dosificador puede aumentar la liberación de fármaco en los pulmones. Debe advertirse que este hecho podría potencialmente conducir a un aumento del riesgo de aparición de efectos adversos sistémicos. Los beneficios del tratamiento con propionato de lluticasona por via inhalatoria deberían reducir la necesidad de administrar esteroides por vía oral, pero los pacientes transteridos que
recibian terapia con esteroides por vía oral pueden seguir estando en situación de riesgo de alteración de la reserva corticosuraran nal durante un tiempo considerable. Los pacientes que han requerido una terapia corticosteroidea de emergencia con dosis altas en el pasado, pueden también estar en situación de riesgo. Esta posibilidad de alteración residual deberá siempre tenerse en cuenta en situaciones de emergencia y electivas que puedan producir estrés, aumentar de manera considerable las concentraciones de propionato de fluticasona en plasma. Por lo tanto, debería evitarse el uso concomitante de propionato de fluticasona y ritonavir, a menos que el beneficio potencial para el paciente sea mayor que el riesgo de aparición de efectos secundarios sistémicos de tipo corticosteroide. También hay un aumento del riesgo de que aparezcan efectos adversos sistémicos cuando se combina el propionato de fluticasona
con otros inhibidores portentes del cYP3A (Ver apartado de Interacción con otros medicamentos y otras formas de interacción). Interacción con otros medicamentos y otras formas de interacción. Deberá evitarse la utilización de
beta blogueantes tanto selectivos como no selectivos en pacientes con asma, a menos que haya razones que obliguen a ello. El uso simultáneo de otros fármacos que contengan agonistas beta adrenérgicos puede tener un efecto potencialmente aditivo. En circunstancias normales, se obtienen baias concentraciones plasmáticas de propionato de fluticasona después de la inhalación, debido a un intenso metabolismo de primer paso hepático y a un alto aclaramiento sistémico producido por el citocromo P450 3 A4 en el intestino e hígado. Por lo tanto, es improbable que se produzcan interacciones medicamentosas clínicamente significativas en las que intervenga el propionato de

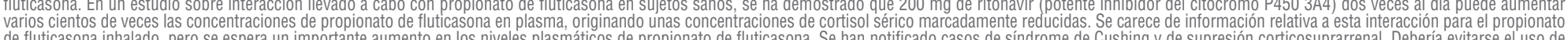
de fluticasona inhalado, pero se espera un importante aumento en los niveles plasmáticos de propionato de fluticasona. Se han notificado casos de sindrome de Cushing y de supresion corticosuprarrenal. Debería evitarse el uso de inhibidor ligeramente menos potente del CYP3A. produjo un aumento de la exposición a propionato de fluticasona tras una única inhalación del $150 \%$. Esto produjo una mayor reducción de los niveles de cortisol plasmático en el riesgo de efectos adversos sistémicos. Se recomienda tener precaución y evitar, en la medida de lo posible, el tratamiento a largo plazo con éstos fármacos. Embarazo y lactancia. No existen datos suficientes sobre el uso de

\begin{tabular}{|l|l|l|}
\hline Clasificación de órganos & Efecto adverso & Frecuencia \\
\hline Infecciones e infestaciones & Candidiasis en la boca y garganta & Frecuentes \\
\hline $\begin{array}{l}\text { Trastornos del sistema } \\
\text { inmunológico }\end{array}$ & $\begin{array}{l}\text { Reacciones de hipersensibilidad con las siguientes } \\
\text { manifestaciones: } \\
\text { Reacciones de hipersensibilidad cutánea } \\
\text { Angioedema (principalmente edema facial y y } \\
\text { orofaríngeo), síntomas respiratorios (disnea y/o } \\
\text { broncoespamo) y reacciones anafilácticas }\end{array}$ & $\begin{array}{l}\text { Poco frecuentes } \\
\text { Muy raros }\end{array}$ \\
\hline Trastornos endocrinos & $\begin{array}{l}\text { Supresión corticosuprarrenal, retraso en el crecimiento } \\
\text { de niños y adolescentes, disminución de la densidad } \\
\text { mineral del hueso, cataratas y glaucoma. }\end{array}$ & Muy raros \\
\hline Trastornos del sistema nervioso & $\begin{array}{l}\text { Cefalea } \\
\text { Temblor }\end{array}$ & $\begin{array}{l}\text { *Muy frecuente } \\
\text { Frecuente }\end{array}$ \\
\hline Trastornos cardiacos & $\begin{array}{l}\text { Palpitaciones } \\
\text { Taquicardia } \\
\text { Arritmias cardíacas (incluyendo fibrilación auricular, } \\
\text { taquicardia supraventricular y extrasístoles) }\end{array}$ & $\begin{array}{l}\text { Frecuentes } \\
\text { Poco frecuente }\end{array}$ \\
Muy raras \\
\hline $\begin{array}{l}\text { Trastornos respiratorios, } \\
\text { torácicos y mediastínicos }\end{array}$ & $\begin{array}{l}\text { Irritación de garganta } \\
\text { Ronquera / disfonía } \\
\text { Broncoespasmo paradójico }\end{array}$ & $\begin{array}{l}\text { Frecuente } \\
\text { Frecuentes } \\
\text { Muy raro }\end{array}$ \\
\hline $\begin{array}{l}\text { Trastonos } \\
\text { musculoesqueléticos y del } \\
\text { tejido conjuntivo }\end{array}$ & $\begin{array}{l}\text { Calambres musculares } \\
\text { Atralgia } \\
\text { Mialgia }\end{array}$ & $\begin{array}{l}\text { Frecuentes } \\
\text { Muy rara } \\
\text { Muy rara }\end{array}$ \\
\hline
\end{tabular}
de agonistas del receptor beta 2 adrenérgico y glucocorticosteroides. Sólo se debe considerar la administración de Seretide embarazadas se debe utilizar la dosis mínima eficaz de propionato de fluticasona requerida para mantener un control adecuado del asma. No se dispone de datos relativos a la leche humana. En ratas, tanto salmeterol como propionato de de lactancia si el beneficio esperado para la madre es superior al posible riesgo para el niño. Efectos sobre la capacidad para conducir y utilizar máquinas. No se han realizado estudios sobre la capacidad para conducir y utilizar máquinas. Reacciones adversas. Como Seretide contiene salmeterol y propionato de fluticasona, es de esperar que aparezcan reacciones adversas de naturaleza y gravedad similares a las atribuidas a cada uno de los compuestos. No aparecen
reacciones adversas adicionales tras la administración conjunta de los dos compuestos. A continuación se indican los frecuencias se han definido de la siguiente forma: muy frecuentes $(\geq 1 / 10)$, frecuentes $(\geq 1 / 100 \mathrm{y}<1 / 10)$, poco frecuentes $(\geq 1 / 1.000$ y $<1 / 100)$, raras $(>1 / 10.000$ y $<1 / 1.000)$ y muy raras $(<1 / 10.000)$, incluyendo notificaciones aisladas. Las reacciones muy frecuentes, frecuentes y poco frecuentes se han identificado generalmente a partir de los datos de ensayos
clínicos. No se ha tenido en cuenta la incidencia en el caso del placebo. Los acontecimientos myy raros proceden
ceneralmente de datos notificados espontáneamente postcomercialización. Se han comunicado efectos secundarios generalmente de datos notificados espontáneamente postcomercialización. Se han comunicado efectos secundarios farmacológicos de un tratamiento con un agonista beta-2, tales como temblor, palpitaciones y cefalea, pero tienden a seer transitorios y disminuyen con una terapia regular. Debido al componente propionato de fluticasona, algunos pacientes
pueden padecer ronquera y candidiasis (afta) en boca y garganta. Tanto la ronquera como la incidencia de candidiasis pueden aliviarse haciendo gargarismos con agua tras utilizar este producto. La candidiasis sintomática puede tratarse mediante una terapia antifúngica tópica mientras se continúa el tratamiento con Seretide. Los posibles efectos sistémicos mineral del hueso, cataratas y y glaucoma (ver el apartado de Advertencias y y precauciones especiales de empleo). Como
sucede con cualquier otra terapia administrada por vía inhalatoria, puede aparecer broncoespasmo paradóijco (ver el
apartado de Advertencias y precauciones especiales de empleo). Sobredosis. No se dispone de datos procedentes de apartado de Advertencias y precauciones especiales de empleo). Sobredosis. No se dispone de datos procedentes de con ambos fármacos: Aguda: La inhalación de forma aguda de dosis de propionato de fluticasona, superiores a las
recomendadas, puede conducir a una supresión temporal de la función corticosuprarrenal. Esto no hace necesario tomar ninguna acción de emergencia ya que la función corticosuprarrenal se recupera en algunos días, como se comprueba en con una posología adecuada para el control de los sintomas (ver el apartado de Advertencias
de empleo). PERIODO DE VALIDEZ Y PRECAUCIONES ESPECIALES DE CONSERVACION: de la luz solar directa. Seretide
CONTENIDO DEL RECIPIENTE: $S$ STEt

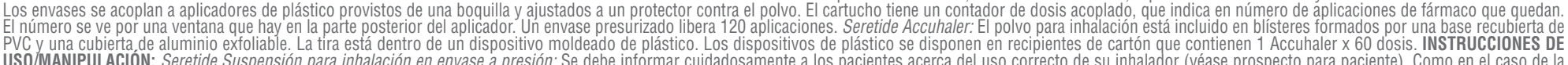
mayoría de los medicamentos administrados por vía inhalatoria dispuestos en envases presurizados, puede disminuir el efecto terapéutico si el envase se enfría. El envase no debe ser perforado, roto o quemado aun cuando

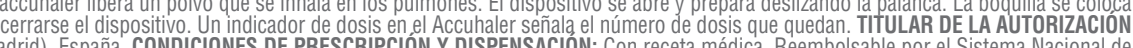

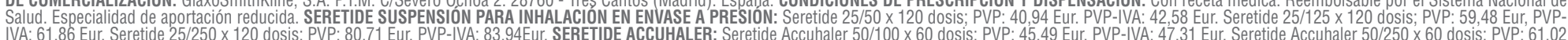
Eur, PVP-IVA: 63,46 Eur. Seretide Accuhaler 50/500 x 60 dosis; PVP: 82,37 Eur, PVP-IVA: 85,66 Eur. Para mayor información consultar la Ficha técnica completa del producto. 
TABLA II

\begin{tabular}{c} 
MEDIDAS DE INTERVENCIÓN PARA MEJORAR LA \\
INFORMACIÓN DEL PACIENTE AMBULATORIO QUE TIENE \\
QUE REALIZARSE UNA ENDOSCOPIA DIGESTIVA \\
\hline
\end{tabular}

Se adjunta a la solicitud de endoscopia el documento de consentimiento informado, para que se aporte al paciente (en él se informa en que consiste, por qué se pide y complicaciones), también se entrega hoja explicativa con la preparación necesaria previa a la endoscopia

Se entrega hoja informativa al indicar la prueba, en la que se expone tipo de prueba, día que se solicita y lugar donde se va a realizar

Se entregan recomendaciones escritas a los pacientes que se le ha realizado alguna técnica terapeútica

luación durante el mes de noviembre de 2003 a otros 100 pacientes procedentes del ambulatorio.

\section{Análisis estadístico}

Se realiza estudio estadístico univariante y test de la $\chi^{2}$ para comparar dos proporciones (niveles de cumplimiento de cada criterio en las dos evaluaciones). Se considera que hay significación estadística sí p $<0,05$.

\section{RESULTADOS}

Se realiza una primera evaluación a un grupo de pacientes (n1 =100), la edad media fue de $59 \pm 19,32$ años (rango de edad desde 16 a 95 años), (59 mujeres y 41 varones), en la segunda evaluación tras las medidas de mejora se estudia otro grupo de pacientes $(\mathrm{n} 2=100)$, con una edad media de $55 \pm$ 16,43 años, rango (15 a 89 años), (61 mujeres y 39 varones).Tras estimar el grado de cumplimiento de los criterios en la primera evaluación, aplicando el cuestionario de recogida de datos (Tabla I): (C1: Información médico prescriptor. C2: Información escrita antes de la endoscopia. C3: Se da consentimiento al solicitar la endoscopia. C4: Existe consentimiento informado. C5: Información médico que realiza la endoscopia. C6: Se aporta documento recomendaciones tras la endoscopia), se determinan aquellos criterios con más incumplimientos (C6, C3, C1) que acumulan una frecuencia relativa acumulada de incumplimientos del $88,88 \%$ (Fig. 1) (Tabla III), lo que permite dibujar la curva de frecuencia acumulada (Fig. 2). A partir de este gráfico y tras señalar un punto en el que se encuentran el 60-80\% de los incumplimientos encontrados, podemos dirigir la priorización de la intervención a los criterios comprendidos en esos pocos vitales, en nuestro estudio actuamos a nivel de los criterios C6 y C3.

Tras la implantación de las acciones de mejora - Tabla II-, se realiza una segunda evaluación, de forma que podamos evidenciar lo que se ha logrado tras la intervención y comprobar si esa mejora ha sido significativa respecto a la situación inicial. Realizamos una segunda evaluación obteniendo unos resultados que comparamos con la inicial (Fig. 3). El estudio estadístico establece una mejoría significativa $(p<0,01)$ en los criterios C6, C3 y C1 (Tabla IV) (Fig. 4).

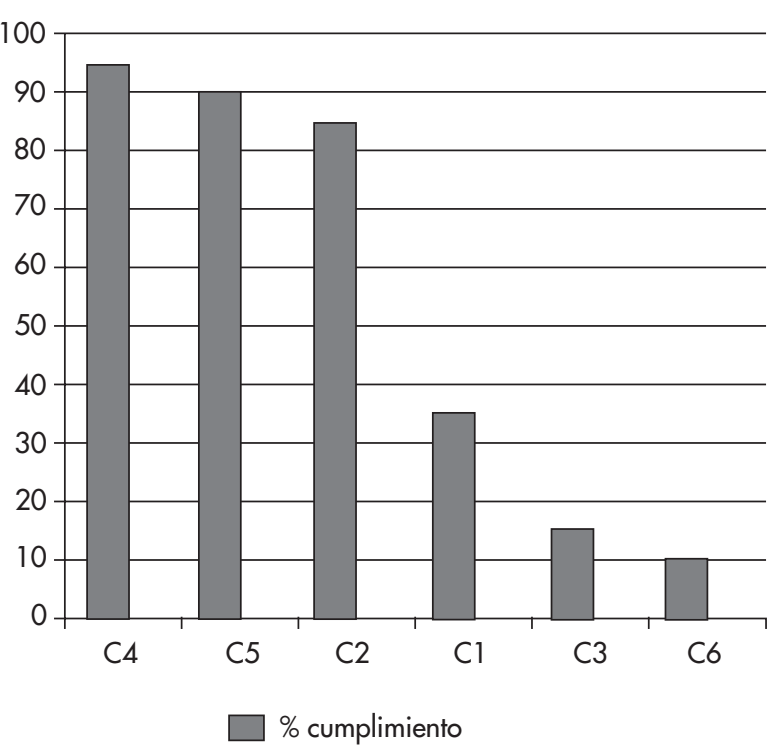

Fig. 1. Porcentaje de cumplimientos de criterios en la primera evaluación. Estimación grado de cumplimiento de los criterios ordenados de mayor a menor cumplimiento evaluados (C1: Información médico prescriptor. C2: Información escrita antes de la endoscopia. C3: Se da consentimiento al solicitar la endoscopia. C4: Existe consentimiento informado. C5: Información médico que realiza la endoscopia. C6: Se aporta documento recomendaciones tras la endoscopia).

\section{TABLA III}

TABLA DE INCUMPLIMIENTOS DE CRITERIOS PRIMERA EVALUACIÓN

\begin{tabular}{lccc}
\hline Criterio & $\begin{array}{c}\text { Frecuencia } \\
\text { absoluta } \\
\text { incumplimientos }\end{array}$ & $\begin{array}{c}\text { Frecuencia } \\
\text { relativa } \\
\text { incumplimientos }\end{array}$ & $\begin{array}{c}\text { Frecuencia relativa } \\
\text { acumulada } \\
\text { incumplimientos }\end{array}$ \\
\hline C6 & 90 & 33,33 & 33,33 \\
C3 & 85 & 31,48 & 64,81 \\
C1 & 65 & 24,07 & 88,88 \\
C2 & 15 & 5,55 & 94,43 \\
C5 & 10 & 3,70 & 98,13 \\
C4 & 5 & 1,85 & 99,98 \\
& Total $=270$ & Total $=100 \%$ &
\end{tabular}

C1: Información médico prescriptor; C2: Información escrita antes de la endoscopia; C3: Se da consentimiento al solicitar la endoscopia; C4: Existe consentimiento informado; C5: Información médico que realiza la endoscopia; C6: Se aporta documento recomendaciones tras la endoscopia.

\section{DISCUSIÓN}

Este estudio se puso en marcha con el objetivo principal de evaluar la eficacia de la realización de un programa de evaluación de calidad en endoscopia digestiva, con la finalidad de mejorar la atención médica del paciente.

La evaluación de la calidad en los servicios de salud, comienza con la identificación de una oportunidad de mejora en 


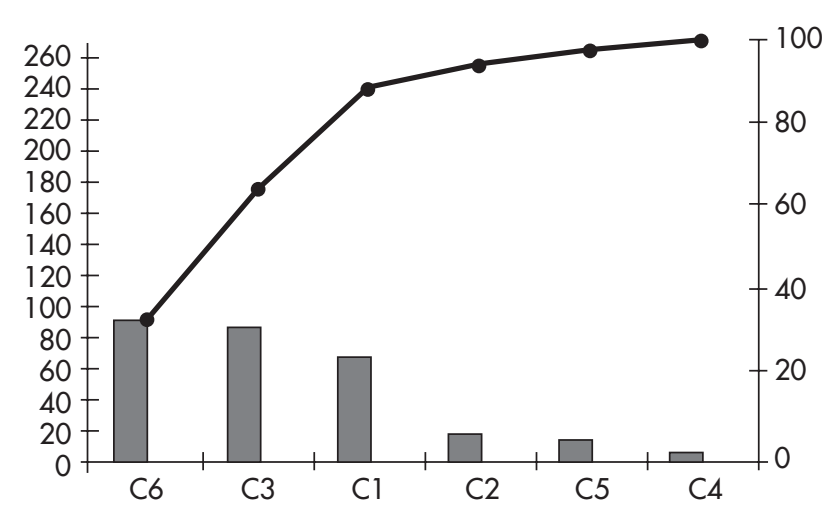

№ total de incumplimientos

Fig. 2. Las barras corresponden al $n^{\circ}$ absoluto de incumplimientos, mientras que la curva representa el porcentaje acumulado de incumplimientos.

algún aspecto de los servicios que se están ofreciendo, este problema puede ser asistencial, organizativo, de satisfacción o de información. El objetivo es, elevar el nivel de calidad del aspecto a evaluar a los niveles máximos que permite el contexto en el que se prestan los servicios. Los ciclos de mejora son una actividad imprescindible en los programas de gestión de la calidad.

Por tanto la intención es aprovechar la oportunidad de mejora para solucionar el problema identificado. Para ello, el curso normal de actividades es:

- Analizar el problema a la luz de los conocimientos que se tenga sobre sus posibles causas.

-Explicitar los criterios, requisitos o especificaciones que nos sirvan para medir el nivel de calidad.

- Diseñar y realizar el estudio.

- Diseñar e implantar la intervención oportuna, cuyo efecto en la calidad ha de ser medido reevaluando de nuevo el tema en estudio (1-4).

Nuestro objetivo era mejorar una situación fundamental en la actividad clínica asistencial, que es el nivel de información que tienen los pacientes antes de una endoscopia digestiva y posteriormente a la misma, pero esta situación es aplicable a otras pruebas diagnóstico-terapeúticas en medicina.

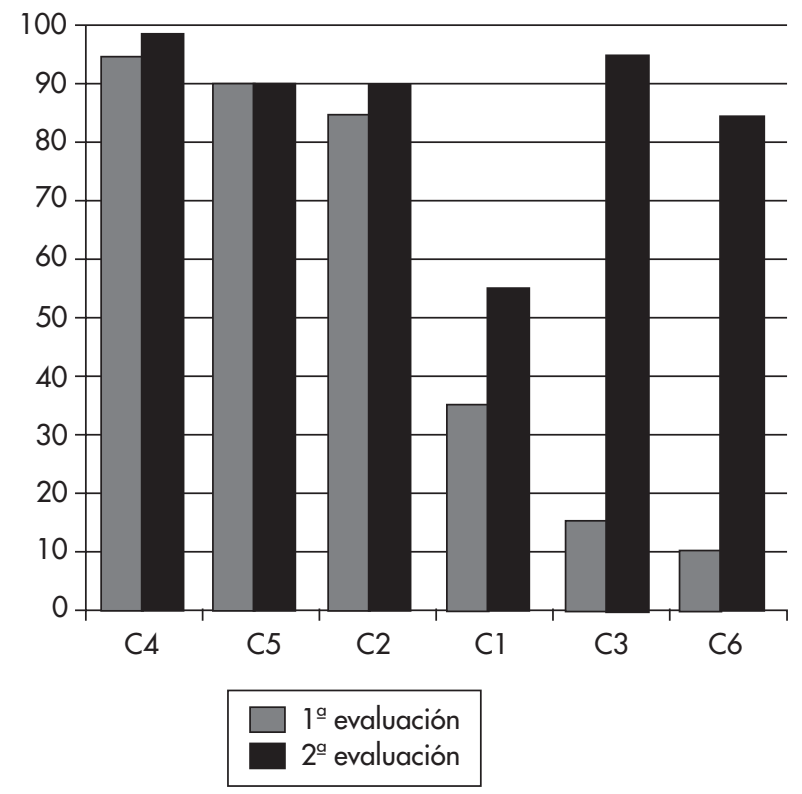

Fig. 3. Comparación porcentajes de cumplimiento de las dos evaluaciones (C1: Información médico prescriptor. C2: Información escrita antes de la endoscopia. C3: Se da consentimiento al solicitar la endoscopia. C4: Existe consentimiento informado. C5: Información médico que realiza la endoscopia. C6: Se aporta documento recomendaciones tras la endoscopia).

Las relaciones entre el médico y paciente se han basado desde hace más de 24 siglos en los principios morales enseñados por Hipócrates: "Estableceré el régimen de mis enfermos de la manera que les sea más provechosa, según mis facultades y a mi entender, evitando todo mal y toda injusticia”. Así pues, estos principios están basados en el respeto que el médico ha de tener por los pacientes, buscando en todo momento el bien de los enfermos.

El médico busca siempre reivindicar la dignidad humana, conocedor del hecho de que su función no siempre es la de curar sino en muchos casos consolar y aliviar el sufrimiento de los enfermos. Esta relación entre el médico y sus pacientes fue siempre directa, buscando el alivio de la enfermedad, sin intermediarios, esperando la mejoría o el consuelo del pacien-

TABLA IV

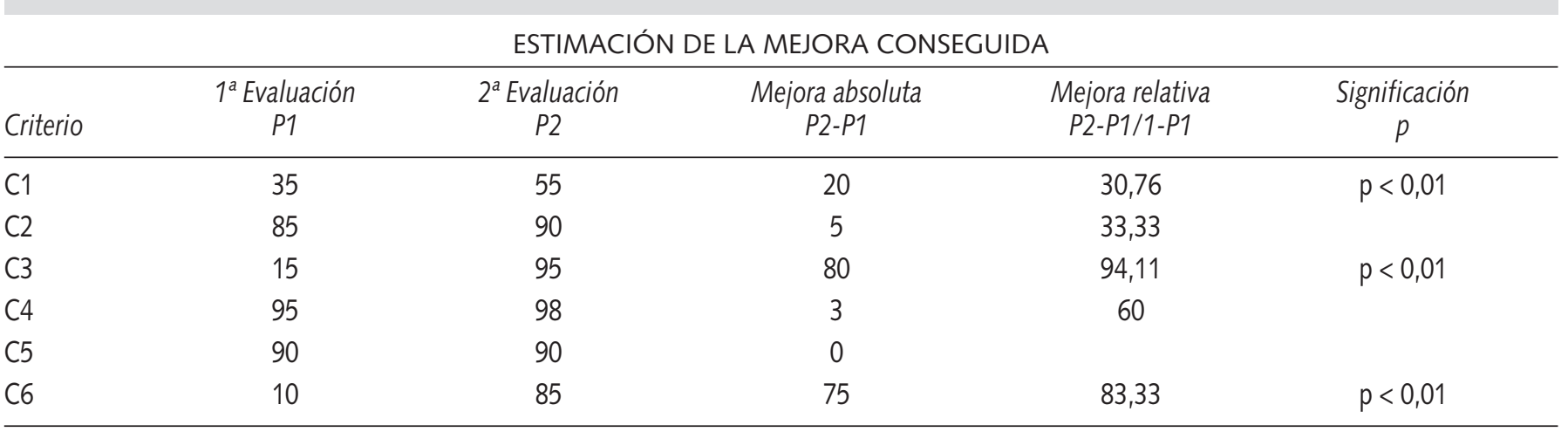

P1 (cumplimiento primera evaluación), P2 (cumplimiento segunda evaluación). 


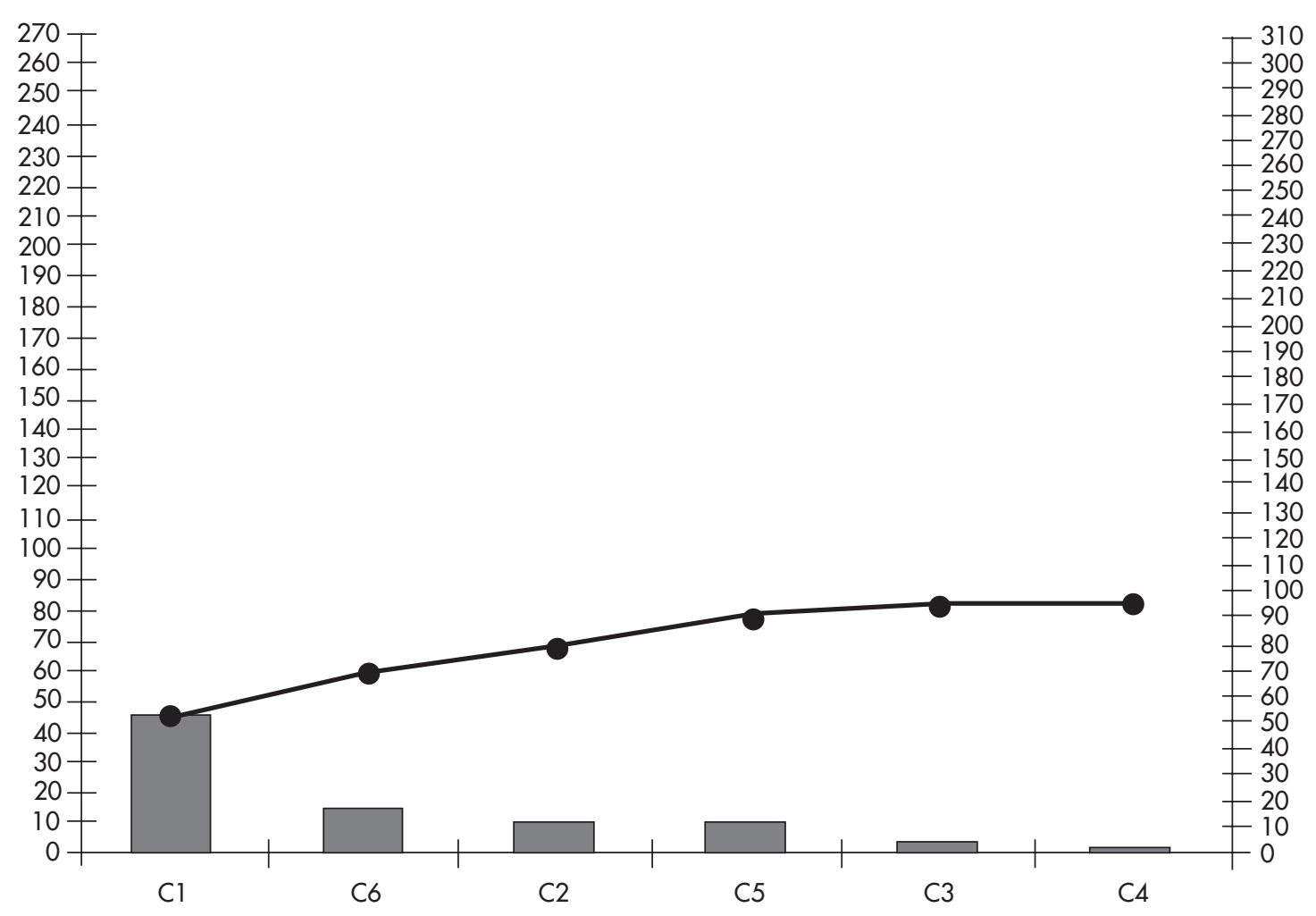

Fig. 4. Tras la reevaluación, por encima de la curva se aprecia la mejora obtenida.

te lo que constituye el “Acto Médico”. Hasta no hace mucho tiempo, el médico conocía el arte y la ciencia y por lo tanto decidía lo que debía hacerse, pensando que era lo mejor para el paciente, en este contexto el paciente no tenía capacidad de decidir, ni siquiera influir lo que se iba a realizar sobre su cuerpo. Sin embargo con el avance de las comunicaciones, los pacientes se hicieron partícipes de los conocimientos, lo que ha dado lugar a un nuevo marco en las relaciones entre los profesionales de la salud y los pacientes que amplía el esquema hipocrático, dándole reconocimiento máximo al paciente como ser autónomo y dueño de sus decisiones, al hacer énfasis en los cuatro principios fundamentales del enfermo que son: el derecho a la vida, el derecho a la asistencia de la salud, el derecho a ser informado de su proceso y el derecho a una muerte digna (5).

En nuestro país el derecho a la información viene regulado por la Ley 41/2002, de 14 de noviembre, que regula la autonomía del paciente y los derechos y obligaciones en materia de información y documentación clínica. En ella se establece en el capítulo II (artículo 4), el derecho a la información asistencial: "Los pacientes tienen derecho a conocer, con motivo de cualquier actuación en el ámbito de la salud, toda la información disponible sobre la misma”. Este derecho también ha sido incorpo- rado a la Constitución Europea, al incorporar ésta el contenido de la Carta de los Derechos Fundamentales de la Unión, en cuyo artículo 3 se regula el consentimiento informado.

La información a los pacientes aparece directamente vinculada a la dignidad, al derecho a la integridad de la persona y a su libertad. Esta información muchas veces dependerá de la sensibilidad del médico en su relación con el paciente, que tendrá la última palabra.

Nuestra intención ha sido mejorar una parte de la asistencia clínica, las deficiencias encontradas son consecuencia de la excesiva demanda asistencial que impide atender de forma adecuada a todos nuestros pacientes, pero no debe ser esta situación impedimento para intentar mejorar. Resulta indispensable desarrollar cuanto antes estrategias de comunicación que ayuden al paciente a aproximarse al profesional de la medicina y le permita formular con toda confianza cuantas preguntas necesite resolver en torno al proceso del que esté siendo tratado, para lo cual es necesario resolver el proceso de información verbal y escrita con el paciente, mejorando y haciendo accesibles a la comprensión los documentos de consentimiento informado, las hojas de recomendaciones para acudir a la prueba o tras esta y lo más importante la comunicación médico-paciente (6). 


\section{Bibliografía}

1. Nathorst-Boos J, Munck IM, Eckerlund I, Ekfeldt-Sandberg C. An evaluation of the QSP and the QPP: two methods for measuring patient satisfaction. Int J Qual Health Care 2001; 13: 257-64.

2. Saturno PJ. Evaluación y mejora de la calidad en servicios de salud. Conceptos y métodos. Murcia: Consejería de Sanidad y Consumo de la Región de Murcia, 2000.

3. Barr DA, Vergun P. Using a new method of gathering patient satisfaction data to assess the effects of organizational factors on primary care quality. Jt Comm J Qual Improv 2000; 26: 713-23.
4. Ausset S, Bouaziz H, Brosseau M, Kinirons B, Benhamou D. Improvement of information gained from the pre-anaesthetic visit through a quality-assurance programme. Br J Anaesth 2002; 88: 280-3.

5. Martínez-Pereda JM, De Lorenzo R. Los médicos y el nuevo código penal. Editores Médicos. Madrid, 1997.

6. Fleischer DE, al-Kawas F, Benjamin S, Lewis JH, Kidwell J. Prospective evaluation of complications in an endoscopy unit: use of the A/S/G/E quality care guidelines. Gastrointest Endosc 1992; 38 : 411-4. 